\title{
Presentation and Appraisal
}

With the publication of this volume, archaeological parasitology has established the legitimacy of its role in the reconstruction of the past. Though formerly often relegated to the appendix of a site description report, the manuscripts herein represent a dramatic presentation of the potential for the expansion of the database generated by integrating paleoparasitological findings with other archaeological data. And how appropriate that this book should be the product of the Instituto Oswaldo Cruz in Rio de Janeiro whose professional staff has contributed so much to bring this specialty to its present distinguished status.

Readers perusing this text will encounter not only the usual individual case reports (though such basic data will continue to be needed for decades to come), but will be treated also to the antiquity of various parasite diseases, their geography and their history. The application of molecular methodology and other modern laboratory techniques has permitted some authors to test hypotheses regarding evolution of parasites and the resulting current host-parasite relationships. By including parasitological observations in evaluation of the archaeological information extracted during a site excavation, some authors gained epidemiological perspectives that led to recognition of useful additional information about behavior of the studied ancient populations. The range of techniques detailed in this volume extends from the simple gross observations of the form and shape of a coprolite (scatology) to the sophisticated methods of molecular biology. The specimens from which so much information was extracted reached far beyond the usual coprolites to include such unexpected exotics as mites, lice, fleas and amber-embedded insects as well as the larger guinea pigs, dogs, llamas and others. Not to be underestimated is the highly utilitarian value of the bibliographical literature reviews offered for some of these parasites.

Some examples of these generalities are itemized below.

Antiquity of various parasites has been addressed by several authors. Guerra et al. identified mites up to about 11,000 years old in coprolites from a Brazilian cave. Reinhard found fish tapeworm eggs in the mummified bodies of northern Chile's earliest coastal settlers, the Chinchorros. Harter et al. identified Ascaris and Taenia ova in Egyptian mummy wrappings from about $700 \mathrm{BC}$. Bouchet et al. penetrated even deeper into antiquity with their identification of Toxocara canis ova up to a half-million years old in a French cave deposit. Pursuit of the antiquity of sand flies that transmit numerous parasites today led Azar and Nel as well as Andrade Filho and Brazil to the recognition that they have been found in 40 mya (million years ago) Dominican amber and in Lebanese amber about 130 mya. Fossilized ancestors of the warm blood-sucking tabanids (Diptera) that have been considered to be transmitters of several parasitic diseases today can be traced to the Lower Cretaceous Period.

Methodology merits an appropriately significant share of these reports. Chame describes how gross observations of coprolites can predict the genus and sex of its origin (scatology). Conventional methods for microscopic coprolite study are described by Bouchet et al., and Reinhard points out that the smaller than expected Diphyllobothrium ova removed from mummy intestines is probably the result of immature ova from postmortem release of proglottids degenerating within the host gut. Molecular methods involving the amplifying potential of the polymerase chain reaction (PCR) technique have been applied to the firm identification of recovered ova with questionable histologic features by Mayo Iniguez et al. and to dog fleas by Dittmar et al. A modification (Random Amplified Polymorphic DNA: RAPD) was found by Mayo Iniguez to be capable of identifying the presence of Enterobius vermicularis even when ova could not be found microscopically in a coprolite.

Geography of parasites has been expanded by Dittmar and Teegen with the finding of the liver fluke Fasciola hepatica in a human and in associated cattle at a 5000 year old German site. Five different parasites were found by Han et al. in Korea and Reinhard et al. remind us that the triatomid insect vector for American trypanosomiasis (Chagas disease) was present 1200 years ago in Northern Mexico when they identified a profound degree of megacolon in a Chihuahuan mummy.

Paleoepidemiology's effects are noted by numerous authors. Nozais details instructive examples for malaria, leishmaniasis, echinococciasis (hyaditosis), schistosomiasis and filariasis. Reinhard notes the role of human behavior in the distribution of pediculosis capitis among Southern Peru's ancient Chiribaya population, while Santoro et al. define the impact of the Inca's conquest of Northern Chile's low valley populations by noting coprolite parasite changes. Matsui et al. observed that Ascaris sp. did not appear prior to rice agriculture development in Japan. Guerra et al. remind us that the spirochete Borrelia burgdorferi (etiological agent of Lyme disease) has been found in museum tick collections. In addition, ancient mites capable of transmitting infectious agents have been found in association with ancient human remains. Those authors also note that human behavior probably led to colonization of domiciliary environments by these insects.

Evolution aspects are included in studies of numerous presentations. Several of those using molecular methodology discuss the significance of base differences between modern and ancient specimens. The report by Araújo et al. provides an excellent model for perspectives on this topic. 
This volume can be viewed as a clarion call for recognition that paleoparasitology has passed through its initial, descriptive stage and now takes its proper place alongside other subdisciplines that are capable of extracting unique information, generating hypotheses and testing them - a status that will surely be welcomed by the scientific community.

Arthur C Aufderheide Departament of Pathology and Laboratory Medicine School of Medicine, University of Minnesota

Duluth, USA 